\title{
¿Para qué leer a los autores grecorromanos? Una perspectiva coeducativa en la formación del alumnado de secundaria
}

\author{
Datos de contacto: \\ María Pilar Molina-Torres \\ Universidad de Córdoba \\ Pilar.molina@uco.es \\ Luis Alberto Marques Alves \\ Universidade do Porto \\ laalves@letras.up.pt
}

Recibido: 29/04/2020 Aceptado: 20/10/2020

\author{
María Pilar MOLINA-TORRES \\ Luis Alberto MARQUES ALVES
}

\begin{abstract}
RESUMEN
Desde una perspectiva interdisciplinar y curricular, el principal objetivo de esta investigación ha sido analizar el carácter coeducativo e histórico que los escritos grecorromanos transmiten acerca de la mujer y las prácticas diarias que aparecen plasmadas en sus relaciones de género. La muestra la componen 208 alumnos y alumnas de tres centros educativos de titularidad pública, matriculados en tercer curso de Educación Secundaria y en la asignatura optativa de cultura clásica. Para la recogida de información se ha utilizado y validado un cuestionario cuantitativo que se agrupó en tres categorías: (1) el método de enseñanza implementado en la asignatura, (2) las creencias de los estudiantes acerca de la mujer romana en la iconografía educativa, y por último (3) las valoraciones del alumnado con relación a las narrativas de los autores grecorromanos. Del mismo modo, para conocer las percepciones de los alumnos planteamos una investigación que se centró en leer cinco textos históricos y responder a varias preguntas guía que sirvieron para orientar sus dosieres de trabajo. Los resultados arrojaron interesantes reflexiones sobre la situación de inferioridad femenina que se recogen en los textos escritos y las imágenes de los manuales educativos. Como conclusión destacamos la mejora en las habilidades históricas y la construcción personalizada en el aprendizaje de la literatura clásica.
\end{abstract}

PALABRAS CLAVE: aprendizaje activo; coeducación; educación ciudadana; enseñanza de la historia; metodología didáctica. 


\title{
Why read Greco-Roman authors? A coeducational perspective in the training of secondary school students
}

\begin{abstract}
From an interdisciplinary and curricular perspective, the main objective of this research has been to analyze the coeducational and historical character that Greco-Roman writings convey about women and the daily practices that appear in their gender relations. The sample is made up of 208 students from three publicly-owned educational centers, enrolled in the third year of Secondary Education and in the optional subject of classical culture. For the collection of information, a quantitative questionnaire that was grouped into three categories was used and validated: (1) the teaching method implemented in the subject, (2) the beliefs of the students about the Roman woman in educational iconography, and finally (3) the assessments of the students in relation to the narratives of the Greco-Roman authors. In the same way, to know the perceptions of the students we propose an investigation that focused on reading five historical texts and answering several guiding questions that served to guide their work dossiers. The results yielded interesting reflections on the situation of feminine inferiority that are collected in the written texts and the images of the educational manuals. In conclusion we highlight the improvement in historical skills and personalized construction in the learning of classical literature.
\end{abstract}

KEYWORDS: active learning; co-education; civic education; history teaching; didactic methodology.

\section{Introducción}

Al hablar de Historia de las Mujeres identificamos el lugar que la mujer ha tenido para los historiadores tanto en un plano particular en las diversas etapas históricas, como a nivel general en igualdad con el género masculino (Flecha, 2004). No obstante, desligar su presencia histórica de la esfera masculina impediría dar sentido a la relación de ambos mundos (femenino/masculino) y construir la Historia y la enseñanza de las Ciencias Sociales. En esta dirección, los estudiantes requieren estudios integrales de género si quieren reconocer y desarrollar sus habilidades para deconstruir los estereotipos tradicionales y contribuir a la justicia social y la inclusión (Cushman, 2012). Esta formación en género se recoge como uno de los temas prioritarios a cubrir en los próximos años en escuelas e instituciones sociales (Colás y Jiménez, 2006).

El término coeducación se dirige a que mujeres y hombres reciban una educación conjunta (Subirats, 2017). La misma autora (1994) afirma que: "la educación igual no tiene un carácter homogéneo en el tiempo, de modo que "coeducación" no ha designado exactamente el mismo modelo educativo en todas las etapas históricas" (p. 49). Tal y como defienden Bejarano, Martínez y Blanco 
(2019) se deben diseñar pedagogías feministas que se opongan a las desigualdades y apoyen los procesos coeducativos. Además, la transmisión de un modelo femenino y/o masculino en la enseñanza obligatoria española nos aporta representaciones simbólicas de mujeres y hombres que pertenecieron a los sectores sociales destacados (Pagès y García, 2008).

Para Calvino (2015) la escuela debe facilitar la elección para leer a los clásicos, aunque realmente la decisión que cuenta es la que se hace fuera de la escuela. Sin embargo, el lugar de la mujer en la literatura grecorromana continúa siendo un tema problemático y lleno de prejuicios. Esta situación ha motivado que en las dos últimas décadas los estudios de género y su enseñanza de la historia haya dado como resultado numerosos estudios (Blanco, 2008; Sant y Pagés, 2011; D'ascenzo, 2013; González-Vaquerizo y López-Gregoris, 2013; Mustapha, 2013; Arias, Sánchez y Martínez, 2014; López-Navajas, 2014; Sáenz del Castillo, 2015; GómezCarrasco y Gallego, 2016; Ortega, 2018).

Ahora bien, desde una perspectiva histórica es necesario preguntarse si la iconografía y los estudios de género en la actualidad han tenido un papel esencial para mostrar una realidad sociocultural que debe ser conocida y recordada (Molina-Torres, 2016). A este respecto, Gómez-Carrasco y Rodríguez (2014b) apuntan que ese conocimiento debe estar basado en la capacidad de análisis de la realidad social y en la movilización de los saberes de geografía e historia para resolver los fenómenos presentes en su vida cotidiana. Se trata, en definitiva, de enseñar la historia preguntándonos si los acontecimientos del pasado en relación con los roles de género se repiten actualmente. En este sentido, hay que destacar que la escuela debe convertirse en una institución que visibilice las acciones y situaciones históricas de las mujeres rechazando cualquier referencia que impida la igualdad educativa. Tal y como indica Suberviola (2012): "La escuela debe llevar a cabo acciones como revisar el sistema de valores y actitudes que transmite, analizar las discriminaciones históricas por razón de sexo, modificar los contenidos educativos evitando los estereotipos y la invisibilidad de las mujeres" (p. 65).

Asimismo, es un hecho que nuestros estudiantes de tercer curso de Educación Secundaria desconocen las particularidades del mundo clásico quizás, en gran parte, por la falta de proximidad a la herencia grecorromana y a la educación literaria en los centros educativos (Sanjuán, 2016). Al respecto Martín-García (2018) apunta que las obras clásicas se alejan de la esfera adolescente, ya que presentan demasiadas dificultades para su comprensión. Esto se puede conseguir a partir de la formulación de cuestiones reflexivas y críticas que inciten al alumnado a pensar de manera crítica y reflexiva (Prats, 2017). Es más, la enseñanza de la Historia señala la empatía histórica en Educación Secundaria como una competencia propia del aprendizaje del pensamiento histórico. De esta manera, como indican Gudín, Lasala y Iturriaga (2017):

La empatía es clave para que los escolares den sentido a la conducta humana, comprendan por qué eran comunes en el pasado prácticas que hoy parecen 
absurdas y puedan reconstruir las razones por las que algo se hizo valorando las intenciones y su visión de la situación (p. 77).

La enseñanza de la Historia y de la Historia Antigua permite al alumnado alcanzar competencias para aprender a interpretar realidades sociales desde un enfoque histórico y también se nos plantea a los docentes como un reto desde la didáctica de la Historia (Pagès y García, 2008). Es aquí donde nos situamos ante diversas formas de enseñar la Antigüedad y facilitar el cambio de los esquemas tradicionales de enseñanza en secundaria. Por todo ello, es necesario dar prioridad a las competencias educativas sobre los contenidos teóricos (Blömeke et al, 2016). El pensar históricamente no se ciñe a recordar los hechos y acontecimientos del pasado (Gómez-Carrasco, Ortuño y Molina-Puche, 2014a; Gómez-Carrasco y Miralles, 2015a), sino en comprender los medios que se necesitan para establecer estos hechos, su interpretación en el espacio cronológico y contexto geográfico (Seixas y Morton, 2013; Domínguez, 2015).

También es significativo resaltar la invisibilidad femenina en el mundo antiguo y su intencionada materialización en los manuales de texto. Nosei (2007) refiere acerca de la lectura de los contenidos de los libros de texto que lejos de contextualizar para favorecer la comprensión de algún hecho, aumentan los niveles de confusión. Precisamente, se entiende como parcialidad la construcción de un relato histórico que prescinda de ese cincuenta por ciento de la humanidad que son mujeres, o de que se haga referencia a ellas sólo tangencialmente. Esta escasez de personajes históricos femeninos en los materiales escolares esconde un currículo oculto que dificulta la educación en valores del alumnado. A esto debemos añadir la discriminación sexista que cualitativa y cuantitativamente observamos en la escasa presencia femenina y su tratamiento iconográfico en los libros de texto no universitarios, que esconde la contribución de las mujeres al desarrollo histórico y social. Esta deficiencia de contenidos curriculares imposibilita un análisis identitario de la etapa histórica estudiada (García-Luque y Peinado, 2015). Tal y como señalan Molina-Puche, Miralles, Deusdad y Alfageme (2017):

La historia, además de una herramienta para tomar conciencia de la situación social del presente a través del análisis crítico del pasado, se convierte también en un instrumento para transformar la realidad, capacitar al alumnado como ciudadano crítico con su tiempo y capaz de comprometerse con su sociedad y actuar para la mejora de ésta (p. 335).

Estas enseñanzas que deben transmitirse al alumnado de secundaria derivan, fundamentalmente, del continuo proceso que adquieren los profesores de Historia en su desarrollo profesional con dos objetivos: enseñar la Historia y construir identidades. Para Zamudio (2012), sus conocimientos atienden a las experiencias vividas y a las circunstancias sociales e históricas del entorno en el que viven. Por este motivo, para el estudio de las Ciencias Sociales y la Historia es preciso que las dificultades de aprendizaje de los estudiantes no sean una consecuencia de un inadecuado proceso de formación del docente (Aguilar, 2013; González-Rivera y 
Skultety, 2018). Al respecto, Sáiz-Serrano y López (2015) consideran que: “educar históricamente equivale a desarrollar el pensamiento histórico de los estudiantes, y que éste no es un conocimiento intuitivo y natural, sino que requiere un proceso formativo gradual" (p. 89).

Por tanto, las habilidades para interpretar el pasado y la empatía histórica se desarrollan en la competencia propia de pensar históricamente lo que, en suma, favorece en el alumnado una conciencia histórica relacionada con la mujer en la Antigüedad. Ahora bien, para acercar al alumnado a los estereotipos de género de la mujer romana es primordial conocer la relación entre los roles masculinos y los femeninos en la Edad Antigua. Si bien es cierto que las tareas encomendadas a ambos son bien distintas. Mientras que al género masculino se le encarga la defensa y estabilidad del grupo social al que pertenece, la mujer se la relega al hogar y al cuidado de sus hijos, a lo que debemos añadir la realización de las labores domésticas, propias de su género (Colom y Mayoral, 2016). Por esta razón, es necesario trabajar la coeducación en las diferentes etapas educativas, para que los estudiantes conozcan los estereotipos de género y la invisibilidad femenina en la didáctica de la Historia.

\section{Objetivos de la investigación}

El objetivo principal de esta investigación se centra en analizar la Antigüedad y exponer las valoraciones del alumnado en cuanto a los roles desempeñados por ambos géneros. Con relación a este objetivo general se recogen los siguientes objetivos específicos:

(1) comprobar el método activo y/o pasivo de enseñanza adquirido en las clases.

(2) analizar los estereotipos de género de los libros de texto.

(3) observar las semejanzas y diferencias de la mujer romana con la actual.

(4) intercambiar opiniones sobre aquellos aspectos históricos que aún se mantienen hoy día.

(5) contextualizar al alumnado en situaciones y hechos que les hagan reflexionar sobre el pasado.

(6) describir el contexto sociocultural y la intencionada moralidad de los autores grecorromanos.

\section{Método}

\subsection{Población y muestra}

La población objeto de estudio se compuso de 208 estudiantes, adscritos a seis grupos distintos, de tres centros educativos de Enseñanza Secundaria de Andalucía. La muestra quedó formada por un total de 94 hombres (45.2\%) y 114 mujeres (54.8\%), que a finales del tercer trimestre del curso 2018/2019 
estudiaron la asignatura de cultura clásica en tercero de ESO. Las edades de los participantes están comprendidas entre los 14 y 15 años. La muestra que no tuvo un carácter probabilístico seleccionó a aquellos grupos de alumnos en los que los docentes responsables de la asignatura se habían implicado de forma directa en la investigación educativa (Bisquerra, 2004). Otra de las razones para dicha elección es que la mayoría del alumnado al comienzo de nuestro estudio tenía un nivel de conocimiento medio-bajo relacionado con la temática que vamos a analizar, al ser ésta su primera toma de contacto con cuestiones relacionadas con la historia de género.

\subsection{Diseño de la investigación}

La investigación adopta un carácter mixto mediante la aplicación de un cuestionario cuantitativo para la recogida de datos y el análisis de varios textos literarios de autores grecorromanos con cinco preguntas de respuesta abierta. En la elaboración de este tipo de cuestionario se han tenido en cuenta una serie de ítems orientados para que los participantes conozcan los objetivos del estudio, el límite de cuestiones y que éstas se ajusten de lo general a lo más concreto (Feixas, 2006). Para la validación de cada uno de los ítems se contó con tres expertos universitarios de Historia Antigua y cinco docentes de Enseñanza Secundaria de dos centros educativos de titularidad pública.

Con respecto a los textos seleccionados hay que destacar aspectos similares a los del cuestionario, entre los que cabrían citar la estructura de las preguntas, que no se repitan y cuidando también de su dificultad para que el alumnado pueda comprenderlas sin problema (Quivy y Campenhoudt, 2008).

\subsection{Instrumento de recogida de información y tratamiento de datos}

En la recogida de información se utilizó un cuestionario diseñado ad hoc con una escala tipo Likert de cinco valores que abarcan desde 1 que equivalía a totalmente en desacuerdo y 5 a totalmente de acuerdo. La intervención se implementó durante un total de ocho sesiones distribuidas durante cuatro semanas. Este instrumento tuvo carácter anónimo con el fin de que respondieran con naturalidad y sin sentirse identificados. La recogida de información se efectuó en el horario lectivo de los estudiantes y dentro del aula. Los alumnos respondieron a veinte ítems para los que recibieron las explicaciones necesarias sobre su realización y siempre en presencia del tutor o tutora del curso. A su vez el cuestionario (figura 1) se agrupó en tres categorías vinculadas al conocimiento de las fuentes históricas: (1) el método de enseñanza implementado en la asignatura (cultura clásica), (2) las percepciones de los encuestados sobre la mujer romana en la iconografía educativa, y (3) su visión sobre las narrativas de los autores grecorromanos. 


\section{Figura 1}

\section{Cuestionario empleado en la investigación. Fuente: elaboración propia}
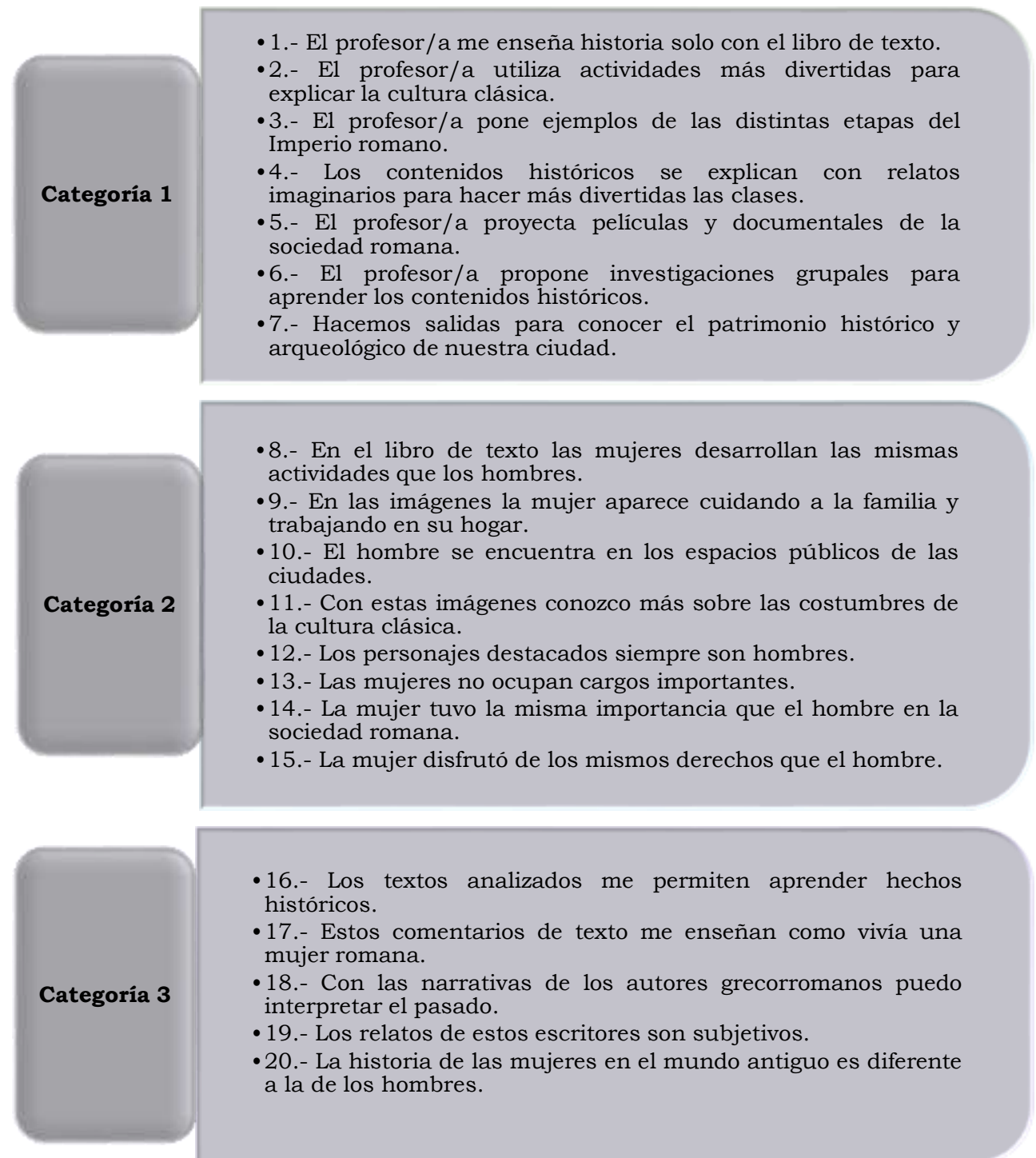

-16.- Los textos analizados me permiten aprender hechos históricos.

-17.- Estos comentarios de texto me enseñan como vivía una mujer romana.

-18.- Con las narrativas de los autores grecorromanos puedo interpretar el pasado.

-19.- Los relatos de estos escritores son subjetivos.

-20.- La historia de las mujeres en el mundo antiguo es diferente a la de los hombres.

Siguiendo el trabajo de Sáiz-Serrano (2014), establecemos una serie de habilidades de utilización de fuentes históricas mediante el planteamiento de una situación de aprendizaje histórico que analizamos para construir evidencias a través de las fuentes del pasado, y por último respondemos e interpretamos las cuestiones planteadas que compilamos en los dosieres finales. En la fase investigativa y la ejecución del portafolio (figura 2), los textos elegidos aportaron una trama literaria activa y sutil de las costumbres romanas, destacando a Plauto 
en Aulularia, Tito Livio en Ab Urbe Condita, Apuleyo en Metamorfosis, Ovidio en Fastos y, por último, Apiano en Historia romana. La lectura de varios fragmentos se realizó en pequeños grupos conformando agrupamientos diferenciados y roles rotativos (coordinador del trabajo grupal, investigador en la búsqueda de información, y encargado de preparar el informe final de la investigación). Para apoyar las argumentaciones de los grupos de investigación, seguirán un guion pautado con cinco interrogantes de carácter abierto:

1.- Describe las actividades que realizan los personajes masculinos $y$ femeninos.

2.- ¿Cuál es su estilo de vida y clase social?

3.- ¿Crees que estas costumbres y situaciones se mantienen en la actualidad?

4.- Sí es así, describe y compara brevemente en qué consisten.

5.- ¿Te parecen "exagerados" los relatos de los autores grecorromanos?

\section{Figura 2}

Fases para analizar los textos literarios. Fuente: elaboración propia

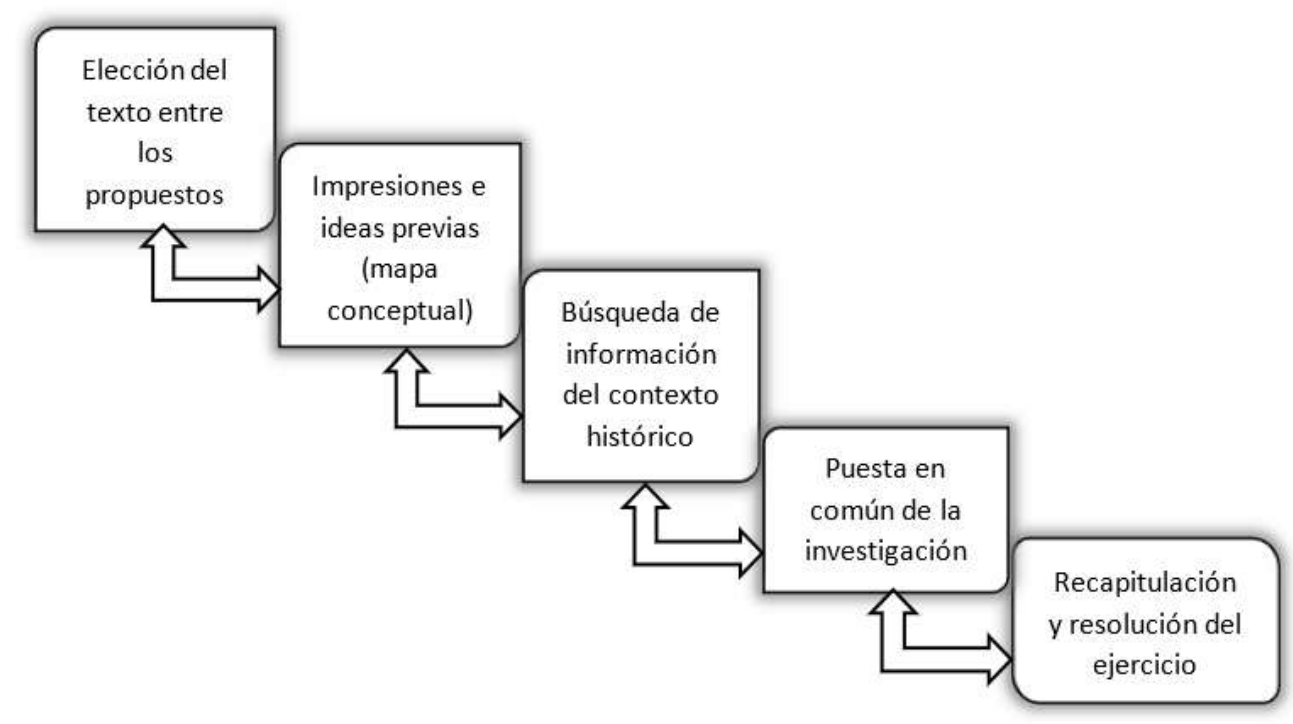

Desde una perspectiva de género, los fragmentos recogidos presentan una visión general de las esferas privada y pública de las ciudades romanas y de los usos culturales del pasado grecolatino (Molina-Torres, 2018). Además, los comentarios de texto ayudarán a reflexionar de manera individual sobre las temáticas analizadas de modo argumentativo y crítico, para plantear las cuestiones esbozadas en gran grupo. Del mismo modo, los textos escogidos anuncian hechos históricos que pueden tener semejanzas y diferencias con la literatura actual (Mata y Villarrubia, 2011). La clasificación literaria se resume a continuación: 
Ritual diario de veneración al Lar Familiaris.

“Dejó un hijo, que es el que vive actualmente aquí en la casa, que es de la misma condición que el padre y el abuelo, y tiene una hija única que no deja pasar un día sin venir a rezarme, me ofrece incienso, vino o lo que sea y me pone coronas de flores. Ella ha sido la causa por la que he hecho encontrar el tesoro a Euclión, su padre, para que la pudiera casar así más fácilmente, si es que quería" (Plauto, Aulularia, 20-26).

Práctica de las supplicationes ante la invasión cartaginesa a Roma.

"El grito de lamento de las matronas se escuchaba por todas partes, no sólo en casas privadas, sino también en los templos. Se arrodillaban en estos y arrastraban sus cabellos despeinados por el suelo, levantando las manos al cielo y lamentándose y suplicando a los dioses para que mantuvieran a la Ciudad de Roma lejos de las manos del enemigo y que preservaran a sus madres e hijos de todo daño o ultraje..." (Livio, Ab Urbe Condita, 26.9)

El culto a Juno Lucina y la Fiesta de Matronalia.

"Mi madre quiere a las mujeres casadas. Las madres vienen en legión a visitarme: esta causa tan piadosa es la que principalmente nos conviene». Traed flores a la diosa; con plantas floridas se regocija esta diosa; ceñid vuestra cabeza con flores tiernas. Decid: «Tú, Lucina, nos diste la luz». Decid: «Atiende tú las plegarias de la parturienta». Y toda la que se halle embarazada, suéltese el pelo y rece para que ella resuelva su parto sin dolor" (Ovidio, Fastos, 3).

Descripción del prototipo de matrona romana.

“(...) de repente me encontré, sin darme cuenta, en el mercado, donde distinguí a una mujer seguida de nutrido cortejo, y me apresuré a ponerme a su altura. El oro que llevaba en joyas y bordados mostraba a las claras que era una verdadera matrona" (Apuleyo, Metamorfosis, 2.2).

\section{Discurso de Hortensia.}

“¿Por qué pagamos tributos, si no compartimos la responsabilidad en los cargos, honores, mandos militares, ni, en absoluto, en el gobierno, por el que lucháis entre vosotros mismos con tan nocivos resultados? Decís: porque es tiempo de guerra..." (Apiano, Historia romana, 4.33).

Se trata, en definitiva, como afirman López-Facal, Miralles, Prats y Gómez (2017): “Investigar en educación consiste en que ese nuevo conocimiento se ocupe de problemas de enseñanza y aprendizaje; y los resultados de la investigación generan nuevas preguntas que permiten seguir avanzando" (p. 14). En este sentido, cabe preguntarse si entre las ventajas del método histórico implementado se favorece la mayor motivación e implicación de los estudiantes en el aprendizaje de la cultura clásica y, de alguna manera, les proporciona la comprensión de una realidad social que indica ciertas diferencias de género en el pasado y el presente. 


\section{Resultados}

Los resultados de esta investigación han dado respuesta a los objetivos planteados al comienzo del estudio. De este modo, hemos obtenido diferentes valoraciones del alumnado basadas en las evidencias históricas analizadas (Rienda, 2016). En un primer lugar, la tabla 1 muestra que en el ítem 1 el 71.2\% de los participantes agradece que su profesor/a explique la asignatura de cultura clásica sin una enseñanza expositiva y memorística, poco motivadora para los alumnos. Sin embargo, podemos afirmar que en la práctica docente los contenidos curriculares se imparten de manera mecánica y repetitiva (Sáiz-Serrano, 2014). Si bien, algunos estudiantes reconocieron que sus clases fueron más interesantes gracias a la utilización de recursos didácticos innovadores y a las salidas didácticas. Esta motivación y las investigaciones que se han planificado en grupo proporcionan un mayor interés por la materia $y$, a su vez, escapan de la rutina docente. Por otra parte, un 53.8\% considera que el docente no ha explicado suficientemente las distintas etapas del Imperio romano (ítem 3). Con este dato se confirma que la explicación previa de este período, antes de implementar la secuencia de actividades vinculadas al mundo clásico, se realizó sin poner énfasis en las causas y las consecuencias que motivaron esta distinción de géneros.

Tabla 1.

Resultados de la categoría uno. Fuente: elaboración propia.

\begin{tabular}{|c|c|c|c|c|c|}
\hline Categoría 1 & $\begin{array}{l}\text { Totalmente } \\
\text { en } \\
\text { desacuerdo }\end{array}$ & $\begin{array}{l}\text { En } \\
\text { desacuerdo }\end{array}$ & $\begin{array}{l}\text { Ni de acuerdo, } \\
\text { ni en } \\
\text { desacuerdo }\end{array}$ & De acuerdo & $\begin{array}{l}\text { Totalmente } \\
\text { de acuerdo }\end{array}$ \\
\hline \multicolumn{6}{|c|}{ Método de enseñanza implementado en la asignatura de cultura clásica. } \\
\hline 1 & $\begin{array}{r}148 \\
(71.2 \%) \\
\end{array}$ & $\begin{array}{r}35 \\
(16.8 \%) \\
\end{array}$ & $\begin{array}{r}14 \\
(6.7 \%) \\
\end{array}$ & $7(3.4 \%)$ & $\begin{array}{c}4 \\
(1.9 \%)\end{array}$ \\
\hline 2 & $\begin{array}{r}15 \\
(7.2 \%) \\
\end{array}$ & $\begin{array}{c}23 \\
(11.1 \%) \\
\end{array}$ & $\begin{array}{r}56 \\
(26.9 \%) \\
\end{array}$ & $\begin{array}{r}32 \\
(15.4 \%) \\
\end{array}$ & $\begin{array}{r}82 \\
(39.4 \%) \\
\end{array}$ \\
\hline 3 & $\begin{array}{r}112 \\
(53.8 \%)\end{array}$ & $\begin{array}{r}43 \\
(20.7 \%)\end{array}$ & $\begin{array}{r}21 \\
(10.1 \%)\end{array}$ & $\begin{array}{r}13 \\
(6.3 \%)\end{array}$ & $\begin{array}{r}19 \\
(9.1 \%)\end{array}$ \\
\hline 4 & $\begin{array}{r}9 \\
(4.3 \%) \\
\end{array}$ & $\begin{array}{r}49 \\
(23.6 \%) \\
\end{array}$ & $\begin{array}{r}37 \\
(17.8 \%) \\
\end{array}$ & $\begin{array}{c}21 \\
(10.1 \%) \\
\end{array}$ & $\begin{array}{r}92 \\
(44.2 \%) \\
\end{array}$ \\
\hline 5 & $\begin{array}{r}4 \\
(1.9 \%)\end{array}$ & $\begin{array}{r}28 \\
(13.5 \%) \\
\end{array}$ & $\begin{array}{r}18 \\
(8.7 \%) \\
\end{array}$ & $\begin{array}{r}23 \\
(11 \%) \\
\end{array}$ & $\begin{array}{r}135 \\
(64.9 \%) \\
\end{array}$ \\
\hline 6 & $\begin{array}{r}16 \\
(7.7 \%) \\
\end{array}$ & $\begin{array}{r}11 \\
(5.3 \%) \\
\end{array}$ & $\begin{array}{r}55 \\
(26.4 \%) \\
\end{array}$ & $\begin{array}{r}28 \\
(13.5 \%) \\
\end{array}$ & $\begin{array}{r}98 \\
(47.1 \%) \\
\end{array}$ \\
\hline 7 & $\begin{array}{r}12 \\
(5.7 \%)\end{array}$ & $\begin{array}{r}13 \\
(6.3 \%)\end{array}$ & $\begin{array}{r}39 \\
(18.8 \%)\end{array}$ & $\begin{array}{r}58 \\
(27.9 \%)\end{array}$ & $\begin{array}{r}86 \\
(41.3 \%) \\
\end{array}$ \\
\hline
\end{tabular}


En cuanto a los resultados de la tabla 2, apuntan a las valoraciones que los estudiantes tienen acerca de las imágenes de la mujer romana que se recogen en sus manuales de texto. Las editoriales analizadas para el estudio fueron Anaya, Edelvives, Oxford, SM y Vicens Vives. Del análisis de los contenidos el alumnado ha podido distinguir las diferencias entre las actividades realizadas por los hombres y las mujeres de la Antigüedad y comprender el tiempo histórico en el que vivieron. A través de estas evidencias, fruto de la interpretación de la iconografía educativa, los estudiantes apuntan con un $43.8 \%$ que la mujer aparece confinada al espacio doméstico y la crianza de los hijos (ítem 9). De hecho, una discreta mayoría se muestra de acuerdo con la inferioridad femenina frente a la masculina en espacios públicos, en el desempeño de honores y cargos administrativos, y en la equiparación de los derechos civiles (ítems 12-15).

\section{Tabla 2}

Resultados de la categoría dos. Fuente: elaboración propia.

\begin{tabular}{|c|c|c|c|c|c|}
\hline Categoría 2 & $\begin{array}{l}\text { Totalment } \\
\text { e en } \\
\text { desacuerd } \\
\text { o }\end{array}$ & $\begin{array}{l}\text { En } \\
\text { desacuerd } \\
\text { o }\end{array}$ & $\begin{array}{l}\text { Ni de } \\
\text { acuerdo, } \\
\text { ni en } \\
\text { desacuerd } \\
\text { o }\end{array}$ & $\begin{array}{l}\text { De } \\
\text { acuerdo }\end{array}$ & $\begin{array}{l}\text { Totalment } \\
\text { e de } \\
\text { acuerdo }\end{array}$ \\
\hline \multicolumn{6}{|c|}{ Percepciones de los estudiantes sobre la mujer romana en la iconografía educativa. } \\
\hline 8 & $34(16.3 \%)$ & $87(41.8 \%)$ & $29(13.9 \%)$ & $19(9.2 \%)$ & $39(18.8 \%)$ \\
\hline 9 & $17(8.2 \%)$ & $12(5.7 \%)$ & $\begin{array}{c}21 \\
(10.1 \%)\end{array}$ & $\begin{array}{c}67 \\
(32.2 \%)\end{array}$ & $\begin{array}{c}91 \\
(43.8 \%)\end{array}$ \\
\hline 10 & $\begin{array}{c}29 \\
(13.9 \%)\end{array}$ & $4(1.9 \%)$ & $\begin{array}{c}41 \\
(19.7 \%)\end{array}$ & $\begin{array}{c}78 \\
(37.6 \%)\end{array}$ & $\begin{array}{c}56 \\
(26.9 \%)\end{array}$ \\
\hline 11 & $\begin{array}{c}42 \\
(20.2 \%)\end{array}$ & $\begin{array}{c}26 \\
(12.5 \%)\end{array}$ & $\begin{array}{c}38 \\
(18.3 \%)\end{array}$ & $\begin{array}{c}29 \\
(13.9 \%)\end{array}$ & $\begin{array}{c}73 \\
(35.1 \%) \\
\end{array}$ \\
\hline 12 & $\begin{array}{c}24 \\
(11.5 \%) \\
\end{array}$ & $\begin{array}{c}34 \\
(16.3 \%) \\
\end{array}$ & $\begin{array}{c}43 \\
(20.7 \%) \\
\end{array}$ & $\begin{array}{c}39 \\
(18.8 \%) \\
\end{array}$ & $\begin{array}{c}68 \\
(32.7 \%) \\
\end{array}$ \\
\hline 13 & $6(2.9 \%)$ & $\begin{array}{c}28 \\
(13.5 \%) \\
\end{array}$ & $\begin{array}{c}61 \\
(29.3 \%) \\
\end{array}$ & $\begin{array}{c}41 \\
(19.7 \%) \\
\end{array}$ & $\begin{array}{c}72 \\
(34.6 \%) \\
\end{array}$ \\
\hline 14 & $\begin{array}{c}76 \\
(36.5 \%) \\
\end{array}$ & $\begin{array}{c}59 \\
(28.4 \%) \\
\end{array}$ & $\begin{array}{c}34 \\
(16.3 \%) \\
\end{array}$ & $\begin{array}{c}21(10.1 \\
\%)\end{array}$ & $18(8.7 \%)$ \\
\hline 15 & $\begin{array}{c}89 \\
(42.8 \%) \\
\end{array}$ & $\begin{array}{c}27 \\
(12.9 \%) \\
\end{array}$ & $\begin{array}{c}49 \\
(23.6 \%) \\
\end{array}$ & $17(8.2 \%)$ & $\begin{array}{c}26 \\
(12.5 \%)\end{array}$ \\
\hline
\end{tabular}

A continuación, los resultados de la tabla 3 muestran la capacidad del alumnado para construir las narrativas de los autores grecorromanos y acercarse 
a la metodología investigativa para representar el pasado histórico. Estas habilidades se muestran en el ítem 18 donde casi el 61.5\% afirma que pueden interpretar el pasado en los relatos históricos analizados. De este modo, la educación histórica del alumnado se encuentra estrechamente vinculada con los contenidos de la materia trabajada y también con su formación cívica (MolinaTorres, 2018). Además, es necesario destacar la subjetividad que impregnan estas fuentes para las que un $51.4 \%$ y un $21.2 \%$ (ítem 19) del alumnado está de acuerdo y totalmente de acuerdo con que la historia femenina se narra con unos roles impuestos social y culturalmente.

De esta forma, un $63.9 \%$ del alumnado está totalmente de acuerdo en que la historia de las mujeres en la Antigüedad es diferente a la de sus coetáneos (ítem 20).

\section{Tabla 3}

Resultados de la categoría tres. Fuente: elaboración propia.

\begin{tabular}{|c|c|l|l|l|l|}
\hline Categoría 3 & $\begin{array}{l}\text { Totalmente } \\
\text { en } \\
\text { desacuerdo }\end{array}$ & $\begin{array}{l}\text { En } \\
\text { desacuerdo }\end{array}$ & $\begin{array}{l}\text { Ni de acuerdo, } \\
\text { ni en } \\
\text { desacuerdo }\end{array}$ & $\begin{array}{l}\text { De } \\
\text { acuerdo }\end{array}$ & $\begin{array}{l}\text { Totalmente } \\
\text { de acuerdo }\end{array}$ \\
\hline \multicolumn{6}{|c|}{ Valoraciones sobre las narrativas de los autores grecorromanos. } \\
\hline 16 & $19(9.1 \%)$ & $26(12.5 \%)$ & $57(27.4 \%)$ & $22(10.6 \%)$ & $84(40.4 \%)$ \\
\hline 17 & $\begin{array}{c}45 \\
(21.6 \%)\end{array}$ & $3(1.5 \%)$ & $\begin{array}{c}67 \\
(32.2 \%)\end{array}$ & $\begin{array}{c}31 \\
(14.9 \%)\end{array}$ & $\begin{array}{c}62 \\
(29.8 \%)\end{array}$ \\
\hline 18 & $6(2.9 \%)$ & $13(6.3 \%)$ & $\begin{array}{c}36 \\
(17.3 \%)\end{array}$ & $\begin{array}{c}25(12 \%) \\
128 \\
(61.5 \%)\end{array}$ \\
\hline 19 & $17(8.2 \%)$ & $12(5.7 \%)$ & $\begin{array}{c}28 \\
(13.5 \%)\end{array}$ & $\begin{array}{c}107 \\
(51.2 \%)\end{array}$ \\
\hline 20 & $25(12 \%)$ & $8(3.8 \%)$ & $3(1.5 \%)$ & $\begin{array}{c}133 \\
(63 \%)\end{array}$ \\
\hline
\end{tabular}




\section{Gráfico 1}

Recuento de respuestas de las tres categorías del cuestionario. Fuente: elaboración propia

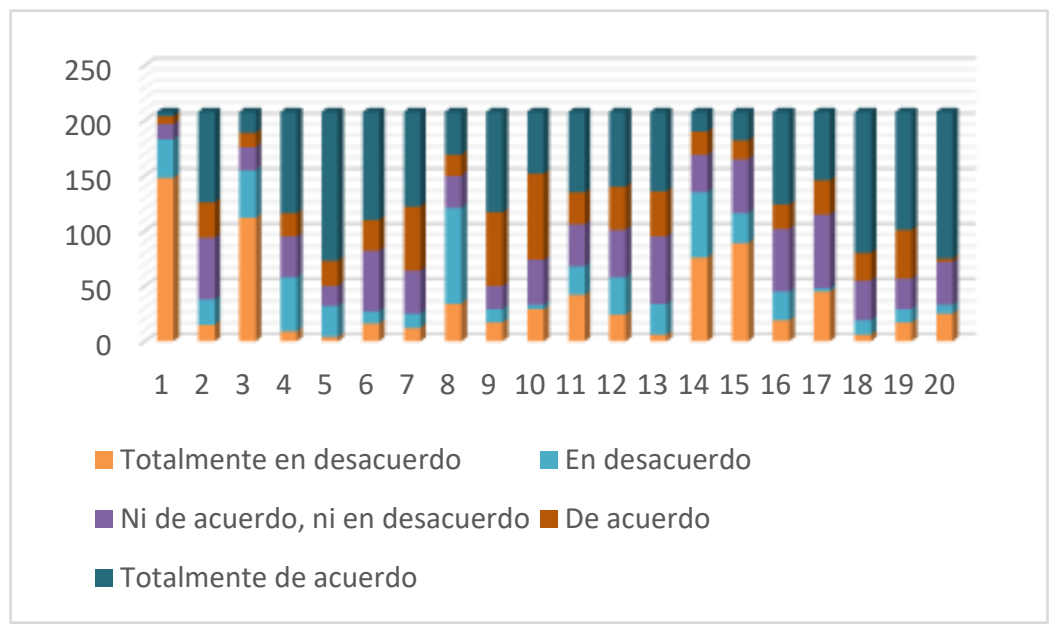

De las opiniones de los estudiantes (gráfico 1) hemos podido comprobar que el alumnado de tercer curso de ESO es consciente de que el estudio de la mujer en la Historia Antigua se interpreta en la iconografía educativa y en la literatura grecorromana como un elemento secundario que relega sus acciones a un espacio privado. No obstante, en las respuestas de los alumnos y las alumnas no pudimos apreciar diferencias de género a la hora de interpretar las cuestiones planteadas. Por otra parte, estos resultados también muestran la repercusión y la implementación de un método de aprendizaje con una materia de historia en la que se utilizan recursos educativos más novedosos y que, en consecuencia, se aleja de la lección magistral y el libro de texto, lo que enriquece la didáctica de la Historia y favorece el descubrimiento del mundo antiguo. Gracias al uso de estas estrategias didácticas, es destacable la implicación del alumnado para analizar, comentar e interpretar la iconografía educativa de género de los textos escolares e identificar a través de los relatos de los escritores grecorromanos los roles masculinos y femeninos, y las evidentes diferenciaciones sociales y económicas asimiladas por ambos géneros.

Por último, a fin de dar respuesta a las cinco cuestiones relacionadas con la lectura de los autores grecorromanos cabe señalar que los resultados fueron muy similares en todos los centros. Para la primera cuestión, debido al volumen de la información recabada y la reflexión de sus actividades investigativas, los detalles aportados sobre las actividades que realizan los personajes masculinos y femeninos de los fragmentos analizados han sido esencialmente generalistas. En estas descripciones no ha sido posible apreciar referencias específicas que indiquen una identificación personal, de género o incluso cívica. De hecho, con frecuencia referían: "en los textos no distingo a más hombres que mujeres y en 
ocasiones no puedo saber lo que hacen ellas..." (E128). También reseñaban la poca importancia que literariamente tenían las mujeres en el mundo clásico, si no eran devotas o pertenecían a familias prestigiosas como fue el caso de Hortensia (E013, E077).

De igual modo, en la segunda cuestión el alumnado expuso: "el estilo de vida de estas mujeres y hombres es diferente, porque no hacen las mismas cosas" (E035); "si una mujer aparece siempre trabajando o rezando, el hombre no aparece nunca haciendo estas actividades... su posición social no es parecida" (E099). Sin embargo, hay opiniones contrapuestas que no siguen las pautas anteriores. Caben destacar algunas interpretaciones, a nuestro juicio, poco coeducativas relacionadas con la posición social de los textos: "no veo diferencias ni económicas, ni tampoco sociales... si una mujer está cuidando del hogar y rezando no tiene ninguna importancia pública en la ciudad, será porque deben estar ahí” (E082); "la mujer debe estar cuidando de su familia y el hombre trabajando fuera de la casa" (E063); "no creo que la posición social influya en que una mujer tenga que estar en un espacio privado (vivienda)... debería ser así, aunque un hombre tenga peor fortuna que una mujer, es él quien ocupa los cargos más importantes y ella pide deseos a los dioses" (E152).

Estas opiniones se relacionan con la cuestión tercera, ya que había un pequeño grupo de alumnos que pensaba que estas situaciones en las que la mujer aparece adscrita al espacio doméstico y el hombre en el público pueden extrapolarse a la sociedad actual. Es aquí donde podemos percibir, aunque no es conveniente generalizar, que los relatos no dejan lugar a dudas del grado de invisibilización de la mujer, si no es para pagar impuestos o realizar ofrendas religiosas. Asimismo, es indiscutible que estos matices no son significativos como norma general en el estudio. De hecho, los alumnos apuntan: "si la mujer reza es porque los dioses le harán caso y a los hombres no...” (E027); "es necesario que una mujer o madre pida lo mejor para los suyos" (E056). En cualquier caso, la cuestión cuarta se complementa con la tercera. Respecto al prototipo de matrona romana piensan: "ahora las mujeres no tienen tantas joyas ni van en coche ni tampoco con esclavos" (E171); "si iban así es porque se lo podían permitir" (E104).

Por último, en cuanto a la veracidad de las reflexiones de los escritores grecorromanos, los participantes relatan la falta de realismo de sus mensajes. Es más, justifican su explicación afirmando: "no todas las mujeres podían gastar tanto dinero, lo que leo se refiere a matronas ricas a las pobres no" (E046); "las mujeres solo rezan o parece que no hacen nada más" (E073); "es muy raro que estén en las casas para pedir a los dioses y también salgan para lo mismo, ¿no hacen nada diferente en las ciudades?" (E206).

\section{Conclusiones}

Es un hecho que el método docente implementado para la enseñanza de la historia y la cultura clásica es un factor clave para educar a los estudiantes en estas disciplinas y, asimismo, formar a ciudadanos con empatía y perspectiva histórica 
(Domínguez, 2015). Los participantes de este estudio constataron con sus respuestas la necesidad de implementar una metodología que les permita aprender Historia Antigua de una manera más dinámica y cercana a la percepción de los acontecimientos históricos. De este modo, no parece lo más adecuado ni conveniente generar una situación de aula centrada exclusivamente en el discurso del profesor. Otro de los aspectos positivos de la investigación ha sido la implicación de los alumnos con un método de indagación como instrumento para el conocimiento de la materia. Sus intervenciones grupales e individuales y los niveles competenciales adquiridos mediante la resolución de problemas, mejoró su interacción con los compañeros y la argumentación de los hechos históricos (Sáiz-Sánchez y Fernández, 2012). A esto último debemos añadir la desconfianza al emprender un proyecto del que desconocían el método de trabajo y los conocimientos teóricos.

Es necesario aclarar que cada categoría no ha sido independiente, de manera que la metodología implementada en la materia va a determinar las reflexiones de los alumnos sobre la iconografía de género, lo que a su vez va a condicionar las percepciones acerca de las narrativas de los autores grecorromanos. Los resultados obtenidos en el cuestionario confirmaron que el uso del libro se compaginó con fuentes iconográficas editoriales y la lectura de textos guiados para estudiar la invisibilidad femenina en la Antigüedad. Podríamos confirmar, por tanto, que la actividad didáctica impartida en la asignatura de cultura clásica necesita de herramientas para comprender el porqué de esta ausencia femenina.

Por su parte, Gómez-Carrasco y Gallego (2016) estudiaron el material iconográfico de los manuales de texto con 152 alumnos de Educación Secundaria. Las opiniones de los alumnos constataron la desigualdad de las imágenes asociadas al género masculino y femenino, y la confirmación de tópicos sexistas en los diferentes ámbitos y actividades que realizaban hombres y mujeres. Otro estudio realizado por Gómez-Carrasco y Tenza (2015b) obtuvo resultados similares. Desde una perspectiva de género, esta insuficiente renovación en los cambios editoriales para la mejora de los libros de texto ha abierto una línea de investigación centrada, fundamentalmente, en la regeneración de las herramientas didácticas para enseñar la historia de las mujeres (Vaíllo, 2016). No obstante, surgen algunas contradicciones cuando intentan identificar en las imágenes de sus manuales la desigualdad social entre hombre y mujer. De hecho, la visión que tienen de la época histórica analizada no evidencia si distinguen entre un espacio público o privado o bien las responsabilidades que se atribuían a ambos sexos. Y así es cómo se transmiten esas funciones diferenciadas por género y valoradas de manera desigual.

En cierto modo, esta dificultad contextual se añade a la constatación de que la mujer ha sido y sigue siendo relegada a las acciones de un espacio privado. Ahora bien, en la Edad Antigua los testimonios iconográficos son parciales lo que causa que esta desigualdad entre hombre y mujer sea un hecho circunstancial que no tiene una explicación de carácter económico o sociocultural (Molina-Torres, 
2018). Por este motivo la representación de las mujeres en el pasado no es una cuestión de género, sino una elección de la editorial en reflejar un periodo histórico concreto con un discurso de carácter masculinizado que se ha confirmado en las respuestas de los alumnos. De ahí que uno de los objetivos de esta investigación no haya sido otro que demostrar esa escasa presencia de lo femenino, que impide a los estudiantes tener un conocimiento general de los hechos históricos de las acciones femeninas que tuvieron la misma repercusión histórica que la de sus homólogos masculinos, a pesar de que la iconografía escolar quiera prescindir de ellas.

En cualquier caso, estas dificultades no resuelven el principal problema de las editoriales al presentar un material iconográfico que no representa por igual la historia de hombres y mujeres. Prueba de ello son las opiniones de los alumnos respondiendo, por un lado, a la necesidad de introducir nuevas imágenes que trabajen la igualdad a través de la historia mientras que, por otro lado, el resultado de la puesta en práctica de las actividades enfocadas al trabajo de la igualdad en el aula promovió una visión reflexiva y positiva para visibilizar a la mujer en el mundo antiguo. Así, y desde un punto de vista didáctico, para el estudio de las mujeres y su historia autoras como López-Navajas (2014) señala:

Aquellos estudios de mujeres han evidenciado no solo la necesidad de recuperar la memoria de las mujeres, sino también la falta de rigor de un relato histórico sesgado e incompleto que normaliza lo que no es más que un elemento de marginación que asienta un orden social excluyente: la invisibilidad de las mujeres en la memoria histórica (p. 286).

Así pues, con estas omisiones se impide tener un conocimiento de las acciones femeninas y los hechos históricos que compartieron con sus homólogos masculinos, lo que nos permite comprobar que la iconografía adaptada por estas editoriales no fomenta la coeducación, ni tampoco ayuda para que la educación en los centros educativos sea de carácter igualitaria (Suberviola, 2012). De la misma manera, se identificó una evidente falta de objetividad en las narrativas de los autores grecorromanos. Por último, los estudiantes se mostraron en desacuerdo con las posturas ideológicas y políticas de los escritores, indicando su rechazo a los estereotipos de género que impregnan los textos literarios y al silencio femenino de las fuentes directas e indirectas que no son materia de revisión en la normativa vigente. 


\section{Referencias}

Aguilar, C. (2013). Género y formación crítica del profesorado: una tarea urgente y pendiente. Revista Interuniversitaria de Formación del Profesorado, 27(3), 177183.

Arias, L., Sánchez, R. y Martínez, A. (2014). Los estereotipos de la historia en el alumnado universitario. En J. Prats, I. Barca y R. López, Historia e identidades culturales (pp. 787-798). Portugal: Universidade do Minho.

Bejarano, M., Martínez, I. y Blanco, M. (2019). Coeducar hoy. Reflexiones desde las pedagogías feministas para la despatriarcalización del curriculum. Tendencias pedagógicas, 34, 37-50.

Bisquerra, R. (2004). Metodología de la investigación educativa. Madrid: La Muralla.

Blanco, N. (2008). Los saberes de las mujeres y la transmisión cultural en los materiales curriculares. Investigación en la Escuela, 65, 11-22.

Blömeke, S., Busse, A., Kaiser, G., König, J. y Sühl, U. (2016). The relation between content-specific and general teacher knowledge and skills. Teaching and Teacher Education, 56, 35-46. DOI: http://dx.doi.org/10.1016/j.tate.2016.02.003

Calvino, I. (2015). Por qué leer los clásicos. Madrid: Siruela.

Colás, P. y Jiménez, R. (2006). Tipos de conciencia de género del profesorado en los contextos escolares. Revista de Educación, 340, 415-444.

Colom, J. y Mayoral, D. (2016). Participación, familia y escuela: la ausencia de lo femenino. Revista Electrónica Interuniversitaria de Formación del Profesorado, 19(1), 113-125. DOI: http://dx.doi.org/10.6018/reifop.19.1.24571

Cushman, P. (2012). You're not a teacher, you're a man: the need for a greater focus on gender studies in teacher education. International Journal of Inclusive Education, $16(8)$, DOI: http://dx.doi.org/10.1080/13603116.2010.516774

775-790.

D'ascenzo, M. (2013). The classical studies secondary school and the construction of national identity in post-unitary Italy. History of Education \& Children's Literature, 8(1), 523-536. DOI: http://dx.doi.org/10.1400/204733

Domínguez, J. (2015). Pensamiento histórico y evaluación de competencias. Barcelona: Graó.

Feixas, M. (2006). Cuestionario para el análisis de la orientación docente del professor universitario. Revista de Investigación Educativa, 24(1), 97-118.

Flecha, C. (2004). Las Mujeres en la historia de la educación. XXI. Revista de educación, 6, 21-34.

García-Luque, A. y Peinado, M. (2015). LOMCE: ¿es posible construir una ciudadanía sin la perspectiva de género? Íber: Didáctica de las Ciencias Sociales, Geografía e Historia, 80, 65-73.

Gómez-Carrasco, C. J., Ortuño, J. y Molina-Puche, S. (2014a). Aprender a pensar históricamente. Retos para la historia en el siglo XXI. Tempo e Argumento, 6(11), 5-27. DOI: http://dx.doi.org/10.5965/2175180306112014005

Gómez-Carrasco, C. J. y Rodríguez, R. A. (2014b). Aprender a enseñar ciencias sociales con métodos de indagación. Los estudios de caso en la formación del profesorado. Revista de Docencia Universitaria, 12(2), 307-325. DOI: http://dx.doi.org/10.6018/reifop.18.3.239001

Gómez-Carrasco, C. J. y Miralles, P. (2015a). ¿Pensar históricamente o memorizar el pasado? La evaluación de los contenidos históricos en la educación obligatoria en España. Revista de Estudios Sociales, 52, 52-48. DOI: http://dx.doi.org/10.7440/res52.2015.04 
Gómez-Carrasco, C. J. y Tenza, S. (2015b). Un género invisible. Análisis de la presencia de las mujeres en los libros de textos de Historia de 4o de la ESO. En A. M. Hernández, C. R. García y J. L. de la Montaña (coords.), Una enseñanza de las ciencias sociales para el futuro: Recursos para trabajar la invisibilidad de personas, lugares y temáticas (pp. 781-791). Extremadura: Universidad de Extremadura.

Gómez-Carrasco, C. J. y Gallego, S. (2016). La pervivencia de estereotipos de género en la enseñanza de la historia. Un estudio a través de libros de texto y las percepciones del alumnado de educación secundaria en España. Revista Electrónica Educare, 20(3), 1-28. DOI: https://doi.org/10.15359/ree.20-3.1

González-Rivera, G. y Skultety, L. (2018). Teacher learning in a combined professional development intervention. Teaching and Teacher Education, 71, 341-354. DOI: https://doi.org/10.1016/j.tate.2018.02.003

González-Vaquerizo, H. y López-Gregoris, R. (2013). Innovaciones en la metodología y la evaluación de la asignatura: transmisión de la cultura clásica. Tendencias pedagógicas, 22, 89-110. DOI: http://dx.doi.org/10.15366/tp

Gudín, E., Lasala, I. y Iturriaga, D. (2017). Didáctica de la competencia histórica en la sociedad digital. Revista de Educación, 375, 61-85. DOI: http://dx.doi.org/10.4438/1988-592X-RE-2016-375-335

López-Facal, R., Miralles, P., Prats, J. y Gómez, C. (2017). Educación histórica y desarrollo de competencias. En R. López, P. Miralles y J. Prats (dirs.), y C. J. Gómez (coord.), Enseñanza de la historia y competencias educativas (pp. 7-22). Barcelona: Graó.

López-Navajas, A. (2014). Análisis de la ausencia de las mujeres en los manuales de la ESO: una genealogía de conocimiento ocultada. Revista de Educación, 363, 114. DOI: http://dx.doi.org/10-4438/1988-592X-RE-2012-363-188

Martín-García, L. (2018). El acceso a la literatura clásica a través de la literatura juvenil contemporánea: estado de la cuestión. Revista de educación de la Universidad de Granada, 95-112. DOI: http://dx.doi.org/10.30827/reugra.v25i0.89

Mata, J. y Villarrubia, A. (2011). La literatura en las aulas: apuntes sobre la educación literaria en la enseñanza secundaria. Textos de didáctica de la lengua y la literatura, 58, 49-59.

Molina-Puche, S., Miralles, P., Deusdad, B. y Alfageme, M. B. (2017). Enseñanza de la historia, creación e identidades y prácticas docentes. Profesorado: revista de currículum y formación del profesorado, 21(2), 331-354.

Molina-Torres, M. P. (2016). La presencia femenina en la enseñanza de las Ciencias Sociales. Reidocrea, 5(27), 278-287.

Molina-Torres, M. P. (2018). Las narrativas de los autores grecorromanos para la enseñanza de la cultura clásica en educación secundaria. En M. I. de Vicente y E. Jiménez (eds.). Investigación e innovación en educación literaria (pp. 179187). Madrid: Síntesis.

Mustapha, A. (2013). Gender and language education research: A review. Journal of Language Teaching and Research, 43, 254-263. DOI: http://dx.doi.org/10.4304/jltr.4.3.454-463

Nosei, M. C. (2007). La enseñanza de la historia en la escuela: una historia de malentendidos. PRAXIS educativa, 11, 74-83.

Ortega, D. (2018). Las mujeres en la historia enseñada: resultados de un programa docente en formación inicial del profesorado. Enseñanza de las Ciencias Sociales, 17, 13-21. DOI: http://dx.doi.org/10.1344/ECCSS2018.17.2 
Pagès, J. y García, T. (2008). La imagen de la Antigüedad en la enseñanza de la historia. En M. J. Castillo (coord.), La Antigüedad en las Artes escénicas y visuales: International Conference "Imagines", The reception of Antiquity in performing and visual Arts (pp. 691-720). Logroño: Universidad de La Rioja.

Prats, J. (2017). Retos y dificultades para la enseñanza de la historia. En P. Sanz, J. M. Molero y D. Rodríguez (eds.), La historia en el aula. Innovación docente y enseñanza de la historia en la educación secundaria (pp. 15-32). España: Milenio.

Quivy, R. y Campenhoudt, L. V. (2008). Manual de investigação em ciências sociais. Lisboa: Gradiva.

Rienda, J. (2016). Límites conceptuales de la composición escrita: alcance de su espacio epistemológico y didáctico. Signa. Revista de Asociación Española de $\begin{array}{lll}\text { Semiótica, 25, 879-901. DOI: } & \end{array}$ http://dx.doi.org/10.5944/signa.vol25.2016.16913

Sáenz del Castillo, A. (2015). ¡La Enseñanza de la Historia sin mujeres! Género, currículum escolar y libros de texto: una relación problemática. Didáctica de las Ciencias Experimentales y Sociales, 29, 43-59.

Sáiz-Sánchez, C. y Fernández, S. (2012). Pensamiento crítico y aprendizaje basado en problemas cotidianos. Revista de Docencia Universitaria, 10(3), 325-346. DOI: http://dx.doi.org/10.4995/redu.2012.6026

Sáiz-Serrano, J. (2014). Fuentes históricas y libros de texto en secundaria: una oportunidad perdida para enseñar competencias de pensamiento histórico. Ensayos. Revista de la Facultad de Educación de Albacete, 29(1), 83-99.

Sáiz-Serrano, J. y López, R. (2015). Competencias y narrativas históricas: el pensamiento histórico de estudiantes y futuros profesores españoles de educación secundaria. Revista de Estudios Sociales, 52, 87-101. DOI: http://dx.doi.org/10.7440/res52.2015.06

Sanjuán, M. (2016). Los factores emocionales en el aprendizaje literario. En J. L. Soler, L. Aparicio, O. Díaz, E. Escolano y A. Rodríguez (coords.), Inteligencia Emocional y Bienestar II. Reflexiones, experiencias profesionales e investigaciones (pp. 156171). Zaragoza: Ediciones Universidad San Jorge.

Sant, E. y Pagés, J. (2011). ¿Por qué las mujeres son invisibles en la enseñanza de la Historia? Historia y memoria, 3, 129-146.

Seixas, P. y Morton, T. (2013). The big six historical thinking concepts. Toronto: Nelson College Indigenous.

Suberviola, I. (2012). Coeducación: Un derecho y un deber del profesorado. REIFOP, 15(3), 59-67. DOI: http://dx.doi.org/10.6018/reifop.22.2.370201

Subirats, M. (1994). Conquistar la igualdad: la coeducación hoy. Revista Iberoamericana de Educación, 6, 49-78.

Subirats, M. (2017). Coeducación, una apuesta por la libertad. Madrid: Octaedro.

Vaíllo, M. (2016). La investigación sobre libros de texto desde la perspectiva de género: ¿hacia la renovación de los materiales didácticos? Tendencias Pedagógicas, 27, 97-124. DOI: http://dx.doi.org/10.15366/tp2016.27.003

Zamudio, J. I. (2012). El conocimiento del profesor que enseña historia. Enseñanza de las Ciencias Sociales: Revista de Investigación, 11, 31-41. 
